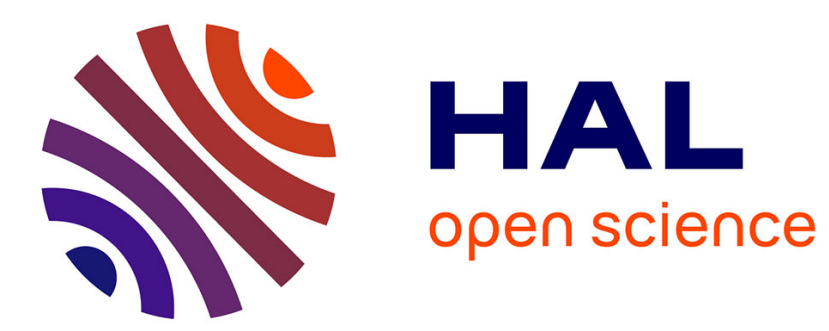

\title{
JOINT DENOISING AND DECOMPRESSION: A PATCH-BASED BAYESIAN APPROACH
}

\author{
Javier Preciozzi, Mario González, Andrés Almansa, Pablo Musé
}

\section{To cite this version:}

Javier Preciozzi, Mario González, Andrés Almansa, Pablo Musé. JOINT DENOISING AND DECOMPRESSION: A PATCH-BASED BAYESIAN APPROACH. ICIP 2017: IEEE International Conference on Image Processing, Sep 2017, Beijing, China. hal-01493635v2

\section{HAL Id: hal-01493635 \\ https://hal.science/hal-01493635v2}

Submitted on 7 Jun 2017

HAL is a multi-disciplinary open access archive for the deposit and dissemination of scientific research documents, whether they are published or not. The documents may come from teaching and research institutions in France or abroad, or from public or private research centers.
L'archive ouverte pluridisciplinaire HAL, est destinée au dépôt et à la diffusion de documents scientifiques de niveau recherche, publiés ou non, émanant des établissements d'enseignement et de recherche français ou étrangers, des laboratoires publics ou privés. 


\title{
JOINT DENOISING AND DECOMPRESSION: A PATCH-BASED BAYESIAN APPROACH
}

\author{
Javier Preciozzi $\quad$ Mario González $\quad$ Andrés Almansa ${ }^{\star} \quad$ Pablo Musé \\ * Facultad de Ingeniería, Universidad de la República, Uruguay \\ ${ }^{\dagger}$ CNRS \& MAP5, Université Paris Descartes, France
}

\begin{abstract}
JPEG and Wavelet compression artifacts leading to Gibbs effects and loss of texture are well known and many restoration solutions exist in the literature. So is denoising, which has occupied the image processing community for decades. However, when a noisy image is compressed, the noisy wavelet coefficients can be assigned to the "wrong" quantization interval, generating artifacts that can have dramatic consequences in products derived from satellite image pairs such as sub-pixel stereo vision and digital terrain elevation models. Despite the fact that the importance of such artifacts in very high resolution satellite imaging has recently been recognized, this restoration problem has been rarely addressed in the literature.

In this work we present a thorough probabilistic analysis of the wavelet outliers phenomenon, and conclude that their probabilistic nature is characterized by a single parameter related to the ratio $q / \sigma$ of the compression rate and the instrumental noise. This analysis provides the conditional probability for a Bayesian MAP estimator, whereas a patch-based local Gaussian prior model is learnt from the corrupted image iteratively, like in state-of-the-art patch-based denoising algorithms, albeit with the additional difficulty of dealing with non-Gaussian noise during the learning process.

The resulting joint denoising and decompression algorithm is experimentally evaluated under realistic conditions. The results show its ability to simultaneously denoise, decompress and remove wavelet outliers better than the available alternatives, both from a quantitative and a qualitative point of view. As expected, the advantage of our method is more evident for large values of $q / \sigma$.
\end{abstract}

Index Terms - Inverse problems in Imaging, Satellite Imaging, Remote Sensing, Joint Denoising and Decompression, Patch-based non-local methods, Bayesian estimation

\section{INTRODUCTION}

Optical satellites with pixel resolution on ground smaller than 1 meter are known as Very High Resolution satellites (VHR). These satellites generate a great amount of information that must be stored on the satellite and transmitted to the ground stations. Both processes are very expensive in terms of data size and this is the main reason for compressing the data on board before sending it to Earth. Thus, compression becomes a key point on the image acquisition pipeline, where wavelet-based compression methods are the standard choice. A great number of satellites follow the specifications defined on the CCSDS recommendations [1], which is based on a CDF 9/7 wavelet

The fourth author performed work while at Télécom ParisTech, France.

This work was partly funded by the French Space Agency (CNES, under grant R\&T 128425/00), by the French Research Agency (ANR), reference ANR-11-LABX-0056-LMH, LabEx LMH, and the Uruguayan Research Agency (ANII). Satellite images courtesy of CNES. CCSDS compression software courtesy of NASA. transform [2]. The compression is then obtained by truncating the wavelet coefficients.

Due to compression three major problems arise: The most evident ones are $(i)$ the presence of Gibbs effects near object boundaries and (ii) the deterioration of micro-textures, both resulting from truncation of wavelet coefficients. The third, less evident one, is the presence of localized artifacts that appear after decompression due to outlier wavelet coefficients. When a coefficient $w(k)$ is affected by acquisition noise, the perturbed coefficient $w_{n}(k)$ may fall in a different quantization interval. The conditions under which these artifacts occur depend on the relationship between the noise power and the size of the quantization intervals (as shown in the experimental section).

Whereas the first two kinds of artifacts are well known and abundantly dealt with in the literature $[3,4]$ the third one is relatively new in the image processing community [5]. Even though wavelet outliers may arise in any imaging device, their importance was first recognized in the context of VHR satellites for two reasons: (a) The absence of an onboard contrast equalization step before compression means that the noise/quantization ratio always reaches critical levels favoring outlier artifacts in some parts of the image; and $(b)$ since VHR satellite images are commonly used as an input for low baseline stereo vision $[6,7]$, it is particularly important to identify and accurately restore this kind of artifacts in order to avoid amplified errors in the final DEM product.

The main objective of this work is to obtain a restored image from the quantized wavelets coefficients. We have decided to face image compression and denoising jointly. This is not a classic approach (denoising and outliers removal are usually performed separately). However, in our opinion this approach enables a better global optimization and a more natural way to model the problem. It also enables the use of the most powerful denoising methods developed so far, those based on local Gaussian models of patches [8,9]. To use these methods, two main issues have to be considered. First, patches are no longer defined on the spatial domain but on the wavelet coefficients domain. Second, the datafit term must take into account both the noise generated by the sensor and the one derived from the quantization process. It will become clear that dealing with these two issues is not trivial. This is the subject of this paper which is organized as follows: In Section 2 we briefly review previous work on satellite imaging restoration and compression artifacts mitigation. Section 3 is devoted to the proposed joint denoising and decompression approach. In Section 4 we present the numerical implementation of the proposed approach. Finally, in Section 5 we apply the joint denoising and decompression method to VHR satellites, which is the case in which the combined effects of instrumental noise and high compression rates are the most significant. The results shows the suitability of the proposed approach. In Section 6 we conclude and discuss possible future work. 


\section{PREVIOUS WORK ON DECOMPRESSION}

The restoration of noisy compressed images requires performing at the same time both denoising and elimination of quantization artifacts. This is a difficult problem whose main features have been described in the previous section. Surprisingly, this problem has not received much attention given the number of acquisition systems that follow this image formation process (satellites but also most imaging systems). In particular, for VHR satellites like Pléiades this problem becomes critical, since accurate generation of disparity maps form stereo pairs and all its derived products (basically 3D reconstruction) are very sensitive to this kind of image degradations.

Two families of state-of-the art approaches to remove quantization noise have been proposed in the literature: dithering methods and variational approaches.

In the dithering methods [5], noise is added before or after quantization in such a way that the joint sensor, quantization and dithering noise is closer to white noise. This kind of techniques is popular among photo-interpreters since it manages to hide outliers in noise, but they decrease the accuracy of subpixel stereo algorithms, thus making them a bad choice for VHR satellite imaging.

As for the variational methods, most methods consist of minimizing the total variation of the restored image with the constraint that quantization errors must fall within the intervals defined by the compressor $[3,10,4]$. These techniques became quite popular to dequantize wavelet coefficients, but in the presence of outliers the quantization interval constraint needs to be relaxed in a robust manner. In Durand and Nikolova [11] this constraint is relaxed by means of a weighted $\ell^{1}$-norm. The adaptation of this method to noisy image decompression was explored in [12]. The results of this modified method are extremely good at neutralizing outliers when these are sufficiently large, sparsely distributed (say no more than $1 \%$ to $5 \%$ of coefficients are outliers) and decorrelated from the background. However, the first two conditions are rarely met in satellite images, where the outlier rates are generally between $5 \%$ and $10 \%$ and may show low amplitudes.

\section{JOINT DENOISING AND DECOMPRESSION METHOD}

\subsection{Notation and problem setting}

From now on we will denote the original, sensed image by $u \in$ $X=L^{2}(\Omega), \Omega$ the image support. The image compression method typically consists of applying a wavelet analysis operator $W$, leading to the wavelets coefficients $w=W u$, followed by a quantization operator $Q$. We denote the quantized wavelet coefficients as $w_{q}=$ $Q w=Q W u$. In this setting, the quantized version of the original image would be obtained as $u_{q}=W^{-1} w_{q}=W^{-1} Q W u$, where $W^{-1}$ denotes the corresponding wavelet synthesis operator.

In a real scenario, the original image $u$ is first contaminated by a random instrumental noise $n$ whose statistics are assumed to be known and will be detailed later, leading to a new image $u_{n}=u+n$. The wavelet coefficients of $u_{n}$ will be denoted by $w_{n}=W u_{n}$, its quantized version by $w_{q n}=Q w_{n}$, and the noisy reconstructed image by $u_{q n}=W^{-1} w_{q n}=W^{-1} Q W u_{n}$.

The difficulty of the problem clearly relies on the fact that $Q$ is non-linear and non invertible, thus making degradation and image statistics intertwined. Therefore, in order to obtain the best possible restoration of $u$ we need to consider the effects of instrumental noise and quantization jointly. In this section we develop a variational model that considers both effects together; the minimization of the corresponding functional will result in the restored version of $u$. As usual, since the problem is ill posed the functional will consist of a prior model and data misfit term. We will call this new method presented here Wavelet Non-Local Bayes (WNLB).

\subsection{Prior model: Patches in the Wavelet domain}

The joint effect of noise and compression results in a highly correlated noise in the spatial domain, with correlations at several scale levels. This renders patch-based MAP estimators intractable in the spatial domain. However, when analyzed in the wavelet domain the noise affecting each wavelet coefficient is nearly decorrelated from the others. For this reason our algorithm works with patches in the wavelet domain, based on the DWT specified on the CCSDS compressor $^{1}$, which is a three-level CDF 9/7 biorthogonal DWT.

Our joint denoising and decompression algorithm follows a coarse to fine strategy. Restoration starts at the coarsest level of the wavelet decomposition; once the wavelet decomposition at this level has been restored, the summary of the next finer level is obtained by the inverse wavelet transform. This process is repeated up to the finest level, from which the final denoised/decompressed image is obtained.

Now, for a given wavelet decomposition level, let $k \in \mathbb{N}^{2}$ denote a spatial location and let $w(k):=\left\{w^{S}(k), w^{H}(k), w^{V}(k), w^{D}(k)\right\}$, where the superscripts $S, H, V, D$ represent the summary, horizontal, vertical and diagonal orientations of the wavelet decomposition. A patch of wavelet coefficients at the scale under consideration centered at $k, P_{k}=p_{k}(w)=\left\{w(k+l) \in \Omega_{p}\right\}$, provides a context of neighboring coefficients $w(k+l)$ that should allow to capture the coefficients correlation structure. The domain $\Omega_{p}$ is a square of $p \times p$ pixels centered at $k$. The optimal size $p$ depends on the signal to noise ratio and will be specified in the experiments.

Inspired by the Non-Local Bayes denoising method [8], the prior model for clean patches is assumed to be a multivariate Gaussian $P_{k} \sim \mathcal{N}\left(\mu_{k}, \Sigma_{k}\right)$, where the mean and covariance parameters $\left(\mu_{k}, \Sigma_{k}\right)$ can be robustly estimated from a set of self-similar noisy patches. It is worth noting that the local Gaussian prior is a well established model for images in the spatial domain, and since the wavelet transform is a linear operator, this model is still suitable for patches in the wavelet domain. The estimation of this Gaussian model is not trivial and details will be discussed in Section 4.1. For the moment we shall assume that $\left(\mu_{k}, \Sigma_{k}\right)$ are known. Then the prior model is (in - log scale)

$$
R_{k}(z):=-\log \mathbb{P}\left[P_{k}=z\right] \propto\left(z-\mu_{k}\right)^{T} \Sigma_{k}^{-1}\left(z-\mu_{k}\right) .
$$

\subsection{Data misfit term}

The data misfit term is obtained by carefully modeling the image degradation process of the wavelet patches. To do so we must first specify the noise model that is adopted here, which is customary in satellite imaging but is general enough to be considered with minor modifications to other optical imaging devices [13].

The image acquisition noise (in the spatial domain) is the superposition of photon noise, electronic noise and quantization noise due to analog-to-digital conversion, and this superposition can be characterized as white Gaussian noise. Despite the instrument point spread function introduces some correlation between neighboring pixels, since its is small the white Gaussian noise approximation with luminance dependent variance $\sigma(x)^{2}=c_{1}^{2}+c_{2} u(x)$ holds reasonable (see [5]). It is worth noting that even in case there could

\footnotetext{
${ }^{1}$ This is the compressor used by the Pleiades VHR satellite; similar considerations apply to the more general JPEG2000 lossy compression method.
} 
exist some deviation from the white noise assumption in the spatial domain, the white noise approximation would make more sense in the wavelets domain. Indeed, the cross-correlation of CDF 9/7 wavelets coefficients is totally negligible [14] with respect of the auto-correlation. The noise variance in the wavelets domain, that we denote by $\sigma_{n}^{2}(k)$, can be readily derived from $\sigma^{2}(x)$ and is given by $\sigma_{n}^{2}(k)=\mathbb{E}\left[(W n(k))^{2}\right]$.

Consider a clean patch $P_{k}=p_{k}(w)$ and its degraded counterpart $\tilde{P}_{k}=p_{k}\left(w_{q n}\right)$. Since the degradation affecting each wavelet coefficient is nearly independent, the multivariate degradation model can be approximated by a product of univariate models

$$
\mathbb{P}\left[\tilde{P}_{k} \mid P_{k}=z\right]=\Pi_{l \in \Omega_{p}} \underbrace{\mathbb{P}\left[w_{q n}(k+l) \mid w(k+l)=z(l)\right]}_{f_{k+l}(z(l))} .
$$

As observed by Zymnis et al. [15] in the context of compressed sensing, each univariate conditional probability $f_{k}(z)$ is a smooth function of $z$ that admits a closed form solution

$$
\begin{aligned}
f_{k}(z) & =\mathbb{P}\left[Q(w(k)+n(k))=w_{q n}(k) \mid w(k)=z\right] \\
& =\mathbb{P}[z+n(k) \in \underbrace{Q^{-1}\left(w_{q n}(k)\right)}_{[a(k), b(k)]}], \quad n(k) \sim \mathcal{N}\left(0, \sigma_{n}^{2}(k)\right) \\
& =\mathbb{P}\left[\mathcal{N}(0,1) \in\left[\frac{a(k)-z}{\sigma_{n}(k)}, \frac{b(k)-z}{\sigma_{n}(k)}\right]\right] \\
& =\Phi\left(\frac{b(k)-z}{\sigma_{n}(k)}\right)-\Phi\left(\frac{a(k)-z}{\sigma_{n}(k)}\right),
\end{aligned}
$$

in terms of the known quantization interval $[a(k), b(k)]$ (here specified by the CCSDS compressor), the noise variance $\sigma_{n}^{2}(k)$ and the erf function $\Phi(x)=\frac{1}{\sqrt{2 \pi}} \int_{-\infty}^{x} e^{-t^{2} / 2} d t$. From the integral form of $\Phi$ it is shown in [15] that the first and second order derivatives of $f_{k}(z)$ also have a closed form, and that $f_{k}$ is a strictly convex function.

To summarize, the conditional probability of an observed patch $\tilde{P}_{k}$, given a clean patch $P_{k}=z$ can be written in $-\log$ scale as

$$
D_{k}(z):=-\log \mathbb{P}\left[\tilde{P}_{k} \mid P_{k}=z\right]=\sum_{l \in \Omega_{p}}-\log f_{k+l}(z(l)) .
$$

\section{NUMERICAL IMPLEMENTATION}

From the previous section, in virtue of (1) and (2) our Bayesian MAP estimator leads to the following optimization problem

$$
\hat{P}_{k}=\arg \max _{z} \mathbb{P}\left[P_{k}=z \mid \tilde{P}_{k}\right]=\arg \min _{z}\left\{D_{k}(z)+R_{k}(z)\right\},
$$

where the regularization term $R_{k}$ is quadratic and the degradation model $D_{k}$ is strictly convex with known gradient and diagonal Hessian. Such a problem can be solved with Newton-type methods.

\subsection{Local Gaussian model estimation}

The previous method requires to estimate a local Gaussian model $\left(\mu_{k}, \Sigma_{k}\right)$ from a set of nearest neighbors $N_{k}=\left\{\tilde{P_{m}}\right\}$ such that $d\left(\tilde{P}_{k}, \tilde{P_{m}}\right)<\delta$. This set of patches are used to estimate the sample mean and covariances of the local Gaussian models. The metric used to compare patches should be robust to the noise present in the images; the covariance estimation may be ill-posed and may therefore

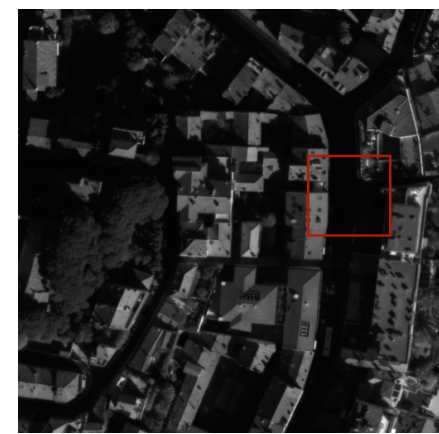

(a) Cannes

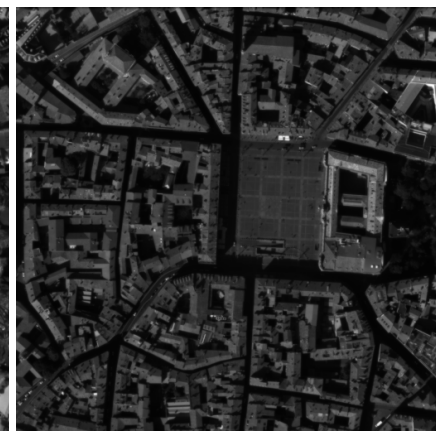

(b) St. Michel
Fig. 1: Images used for the experiments. Both images are 12 bits Pléiades images. The zone delimited by the red rectangle in the Cannes image is the one highlighted in Figure 2.

require regularization techniques. These issues also occur in NLBayes (with several additional challenges that are discussed below); we refer the reader to [8] for the technical details.

\subsection{Weighted Aggregation}

The minimisation problem stated in the previous section is based on patches. That means that the solution for each minimisation problem is a patch. Since each wavelet coefficient belongs to several patches, we have at our disposal several estimators of the same wavelet coefficient to be aggregated. In NLBayes this aggregation is performed by taking the mean value of all patches at each given pixel. This simple approach does not take into account the confidence of each of the restored patches. As shown by Kevrann [16], better results are obtained by weighting each patch contribution with this information: $\hat{w}(k)=\sum_{i} \mathbb{P}\left(\hat{P}_{k_{i}}\right) \hat{P}_{k_{i}}\left(k-k_{i}\right)$, where $\mathbb{P}\left(\hat{P}_{k_{i}}\right)=e^{-F_{k}\left(\hat{P}_{k_{i}}\right)}$ is the posterior probability of the estimator $\hat{P}_{k_{i}}$, and $F_{k}\left(\hat{P}_{k_{i}}\right)=$ $D_{k}\left(\hat{P}_{k_{i}}\right)+R_{k}\left(\hat{P}_{k_{i}}\right)$ is the original functional evaluated on $P_{k_{i}}$.

\section{APPLICATION TO VHR SATELLITE IMAGING}

\subsection{Models of sensor noise and CCSDS compressor}

Luminance-dependent noise. In the case of Pléiades images, the noise model is considered Gaussian but with a variance that depends on the luminance, as already described.

Outliers coefficients. When a coefficient $w(k)$ is affected by acquisition noise, the perturbed coefficient $w_{n}(k): Q^{-1}(w(k)) \neq$ $Q^{-1}\left(w_{n}(k)\right)$. This problem leads to artifacts in the original spatial image, that can be seen clearly in Figure 2. This figure only shows the lower part of its 12 bit dynamic range in order to highlight dark areas at the expense of saturation in lighter areas. Observe that such outliers are apparent as such only when the ratio $q / \sigma$ is relatively large, thus making an outlier event relatively rare and isolated. For moderate compression rates like the 2 bpp example in Figure 2 this situation arises only in dark areas where $\sigma$ takes the smallest values. In lighter areas (where $\sigma$ is larger) or for lower compression rates (for instance $4 \mathrm{bpp}$, where $q$ is smaller), the $q / \sigma$ ratio may take very small values. In such a situation outliers are omnipresent, and the degradation statistics approaches those of white Gaussian noise. 


\begin{tabular}{|l|c|c|c|}
\hline Original vs & Noisy Image & NLBayes + VST & WNLB \\
\hline \hline Cannes & $13.79(0.97)$ & $49.69(0.97)$ & $\mathbf{4 9 . 8 3 ( 0 . 9 7 )}$ \\
\hline St. Michel & $50.21(0.98)$ & $50.53(0.99)$ & $\mathbf{5 0 . 7 8 ( 0 . 9 9 )}$ \\
\hline
\end{tabular}

Table 1: Quantitative analysis of the proposed method for different compression ratios (Cannes 2.0 bpp and St. Michel $2.7 \mathrm{bpp}$ ), compared with NLBayes + VST. Results are expressed in PSNR (SSIM between brackets).

\begin{tabular}{|l|c|c|c|c|c|}
\hline$q / \sigma$ quantile & $\mathrm{Q} 1$ & $\mathrm{Q} 2$ & $\mathrm{Q} 3$ & $\mathrm{Q} 4$ & $\mathrm{Q} 5$ \\
& $0.7: 2.3$ & $2.3: 2.7$ & $2.7: 3.2$ & $3.2: 4.1$ & $4.1: 9.5$ \\
\hline Noisy & 50.33 & 50.20 & 50.18 & 50.22 & 50.13 \\
\hline NLBayes & 50.55 & 50.40 & 50.40 & 50.44 & 50.33 \\
WNLB & 50.64 & 50.52 & 50.51 & 50.54 & 50.46 \\
\hline Increment & 0.093 & 0.11 & 0.11 & 0.10 & 0.13 \\
\hline
\end{tabular}

Table 2: Analysis of the performance of the presented method for different $q / \sigma$ ratios. The table shows the PSNR grouped by quartile. The values correspond to St. Michel Image compressed at $2.7 \mathrm{bpp}$.

\subsection{Results}

The results presented in the following illustrate the performance of the proposed method and its main features. Experiments were performed on simulated Pléiades images, with its corresponding noise model and its CCSDS compression method. Performance evaluation of WNLB is evaluated by comparing its results with those obtained by NLBayes. In order to make this comparison fair, we make use of a noise whitening process, known as Variance Stabilization Transform (VST) [17], that enables to partially whiten the noise making it compatible with the white Gaussian noise assumption of NLBayes. Figure 1 shows two 12 bits Pléiades images used for the experiments: one from Cannes and the other from Toulouse (Place St. Michel). In both cases the instrumental noise constants are $c_{1}=1.9224$ and $c_{2}=0.0339$, but the compression ratios are different: Cannes is compressed at $2.0 \mathrm{bpp}$ while St. Michel is compressed at $2.77 \mathrm{bpp}$.

Figure 2 presents the results on the Cannes image. This example clearly shows one of the major problems derived from the compression of noisy images: the presence of wavelet outlier coefficients introduces visual artifacts (see for example the detail corresponding to the noisy image). It is clear that NLBayes cannot completely remove all the outliers, which are still present in the scene. In Table 1 we present the quantitative analysis of the method by comparing the PSNR and SSIM [18] obtained for the proposed method against NLBayes + VST. We also include in the table results for the St. Michel image, where the compression ratio was $2.77 \mathrm{bpp}$, which is the nominal compression ratio for Pléiades images. Finally, in Table 2, we present a quantitative analysis of the proposed method in relation to $q / \sigma$ ratio for the St. Michel image. This analysis allows to distinguish two different regimes: When $q<\sigma$ almost all coefficients contain wavelet outliers induced artifacts and the degradation is very close to white noise. In this situation both WNLB and NLBayes + VST performance similarly. However, when $q \gg \sigma$ artifacts become more isolated events, and the degradation deviates significantly from white Gaussian noise. In this case NLBayes cannot get its full potential, but WNLB performs particularly well.

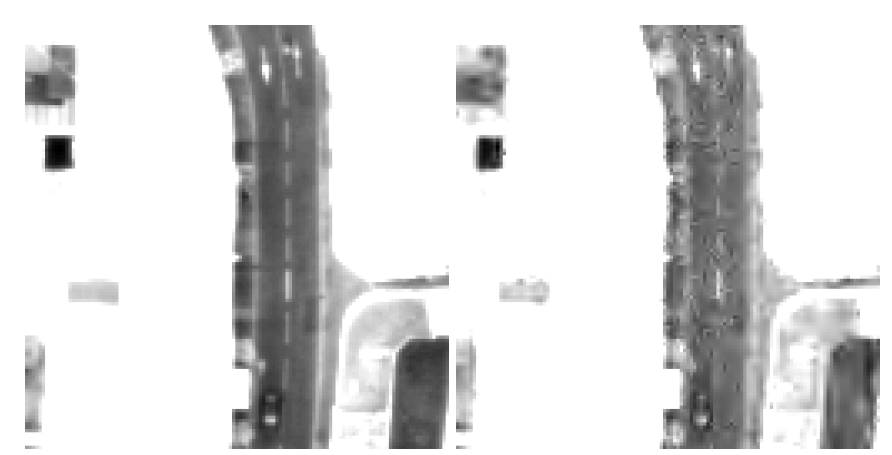

(a) Original Image

(b) Noisy and quantised image

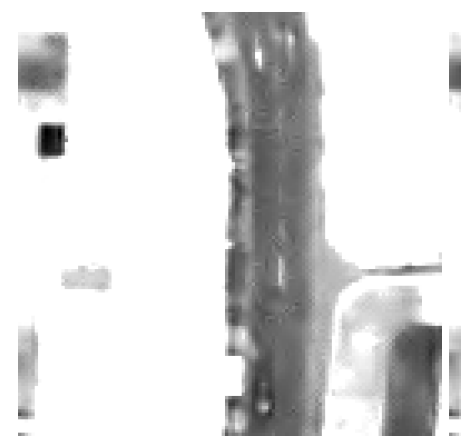

(c) Restored image using NLBayes

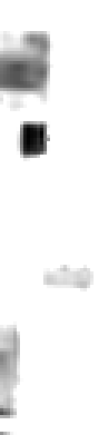

(d) Restored image using WNLB

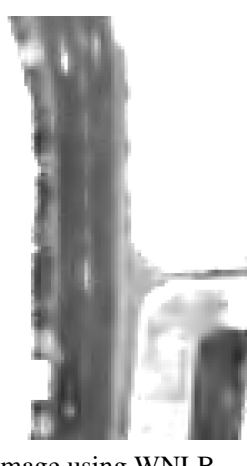

Fig. 2: Detail of the results obtained for an image with luminancedependent local variance and 2 bpp compression ratio. Image range is saturated to view details on the dark regions (in this case, the street).

\section{CONCLUSION}

Wavelet outliers that result from the interaction between noise and compression can be a serious hindrance for stereoscopic vision from VHR satellite images. To correctly deal with this artifacts a joint denoising and decompression approach is required. This work proposes a first patch-based algorithm for this task. Our approach generalizes a state-of-the-art denoising algorithm (NLBayes) that has been adopted by space agencies and professional digital photographers alike. As such its performance is comparable to that of NLBayes as long as compression artifacts are negligible with respect to the noise variance. However the full advantage of our method becomes evident when compression is relatively strong with respect to noise. In the future an extensive calibration of patch sizes as a function of signal-to-noise ratio still has to be performed in order for this method to show its full potential. Other generalizations like including deconvolution are envisioned.

\section{REFERENCES}

[1] Various, "Image data compression - ccsds 122.0-b-1," Tech. Rep., The Consultative Committee for Space Data Systems, 2005.

[2] Albert Cohen, Ingrid Daubechies, and J-C Feauveau, "Biorthogonal bases of compactly supported wavelets," Com- 
munications on pure and applied mathematics, vol. 45, no. 5, pp. 485-560, 1992.

[3] S. Durand and J. Froment, "Reconstruction of wavelet coefficients using total variation minimization," SIAM, Journal on scientific computing, vol. 24, no. 5, pp. 1754-1767, 2003.

[4] P. Weiss, L. Blanc-Feraud, T. Andre, and M. Antonini, "Compression artifacts reduction using variational methods : Algorithms and experimental study," in 2008 IEEE International Conference on Acoustics, Speech and Signal Processing, March 2008, pp. 1173-1176.

[5] Mikael Carlavan, Optimization of the compression/restoration chain for satellite images, Ph.D. thesis, University of Nice Sophia Antipolis, 2013.

[6] Julie Delon and Bernard Rougé, "Small Baseline Stereovision," Journal of Mathematical Imaging and Vision, vol. 28, no. 3, pp. 209-223, jul 2007.

[7] L. Igual, J. Preciozzi, L Garrido, A. Almansa, V. Caselles, and B. Rougé, "Automatic low baseline stereo in urban areas," Inverse Problems and Imaging, vol. 1, no. 2, pp. 319-348, apr 2007.

[8] Marc Lebrun, Antoni Buades, and Jean-Michel Morel, "A nonlocal bayesian image denoising algorithm," SIAM Journal on Imaging Sciences, vol. 6, no. 3, pp. 1665-1688, 2013.

[9] Guoshen Yu, G. Sapiro, and S. Mallat, "Solving inverse problems with piecewise linear estimators: From gaussian mixture models to structured sparsity," Image Processing, IEEE Transactions on, vol. 21, no. 5, pp. 2481-2499, May 2012.

[10] F. Alter, S. Durand, and J. Froment, "Adapted total variation for artifact free decompression of jpeg images," Journal of Math. Imaging and Vision, vol. 23, pp. 199-211, 2005.

[11] Sylvain Durand and Mila Nikolova, "Denoising of frame coefficients using $\ell^{1}$ Data-Fidelity term and Edge-Preserving regularization," SIAM Multiscale Modeling \& Simulation, vol. 6, no. 2, pp. 547-576, 2007.

[12] Javier Preciozzi, Two Restoration Problems In Satellite Imaging, Ph.D. thesis, Universidad de la República, 2016, http://iie.fing.edu.uy/ pmuse/docs/ papers/Thesis20161221.pdf.

[13] Henry Maître, From Photon to Pixel, Wiley, 2015.

[14] Jabran Akhtar, "Optimization of biorthogonal wavelet filters for signal and image compression," M.S. thesis, Hovedoppgave, University of Oslo, 2001.

[15] Argyrios Zymnis, Stephen Boyd, and Emmanuel Candès, "Compressed sensing with quantized measurements," IEEE Signal Process. Lett., vol. 17, no. 2, pp. 149-152, 2010.

[16] Charles Kervrann, "PEWA: patch-based exponentially weighted aggregation for image denoising," in NIPS, 2014, pp. 2150-2158.

[17] M. Lebrun, M. Colom, and J. M. Morel, "The noise clinic: A universal blind denoising algorithm," in 2014 IEEE International Conference on Image Processing (ICIP), Oct 2014, pp. 2674-2678.

[18] Zhou Wang, A. C. Bovik, H. R. Sheikh, and E. P. Simoncelli, "Image quality assessment: from error visibility to structural similarity," IEEE Transactions on Image Processing, vol. 13, no. 4, pp. 600-612, April 2004. 